\title{
Tiers payant à la MediData - eine Verleitung zum Vertragsbruch und ein Stück Verzicht auf unternehmerische Freiheit
}

\author{
H.-W. Leibundgut ${ }^{a}$, B. Berger ${ }^{b}$, P. Baumann ${ }^{c}$
}

Während im System des Tiers garant die versicherte Person dem Leistungserbringer das Honorar schuldet und ihn bezahlt, schuldet beim Tiers payant der Versicherer dem Leistungserbringer das Honorar. MediData preist der hiesigen Ärzteschaft einen Systemwechsel zum Tiers payant an. Wer den Schalmeienklängen des kasseneigenen Intermediärs misstraut, ist gut beraten, wie im folgenden dargelegt wird.

a Dr. med., VR-Präsident Trustcenter PonteNova, Gümligen

b Dr. iur., Advokaturbüro Kellerhals, Bern

c lic. oec., VR-Delegierter NewIndex, Winterthur

Korrespondenz:

Dr. med. Hans-Werner Leibundgut

VR-Präsident PonteNova

Postfach

CH-3073 Gümligen

E-Mail: hans.w.leibundgut@hin.ch
In ihrem Rundschreiben vom 23. Oktober 2004 an die Ärzteschaft weist MediData in einem Postskriptum auf den angeblich problemlosen Wechsel vom Tiers garant auf den Tiers payant hin: «MediPort ermöglicht in den meisten Kantonen den Wechsel vom Tiers garant auf das Tiers payant Abrechnungsverfahren. Profitieren Sie von der schnellen Bezahlung und der Aufhebung der Limitationen durch die Kostenträger.»

Das Schreiben listet weiter sämtliche Kostenträger auf, welche diesen Wechsel akzeptieren. Von den Krankenkassen sind dies: Concordia, CSS, Groupe Mutuel, Helsana, Sanitas, Visana und Wincare.

Wahrscheinlich sind sich die wenigsten Ärztinnen und Ärzte über die Tragweite eines Wechsels in den Tiers payant bewusst. MediData hat auf einen warnenden Artikel («Punktuell» 4/04, Newsletter des TrustCenter PonteNova) mit rechtlichen Schritten gedroht. Wir wollen hier der Ärzteschaft - am Beispiel des Kantons Bern die geltende vertragliche Regelung in Erinnerung rufen und betriebswirtschaftliche Argumente gegen den Tiers payant aufführen.

Der bernische Anschlussvertrag zum Rahmenvertrag TARMED bezeichnet in Art. 11 Abs. 1 den Tiers garant als den vertraglich vereinbarten Abrechnungsmodus. Gemäss Art. 11 Abs. 2 des Anschlussvertrages können bei Vertragsbeginn (1. Januar 2004) bestehende Vereinbarungen über Direktzahlungen (Tiers payant) weitergeführt werden. Wird dagegen nach Vertragsbeginn neu der Tiers payant vereinbart, liegt darin ein Verstoss gegen den Anschlussvertrag (Art. 11 Abs. 1) und damit auch gegen den Rahmenvertrag TARMED.

Daraus folgt, dass die dem bernischen Anschlussvertrag unterstehenden Ärztinnen und Ärzte während der Dauer des Anschlussvertrages und des Rahmenvertrages TARMED grundsätz- lich durch vertragliche Vereinbarung auf den Tiers garant verpflichtet sind.

Selbstverständlich können Krankenkassen und Ärztinnen oder Ärzte auch nach Beginn des Anschlussvertrages bzw. des Rahmenvertrages (1. Januar 2004) auf den Tiers payant wechseln. Ohne den Anschlussvertrag bzw. den Rahmenvertrag zu verletzen, geht dies im Kanton Bern jedoch nur unter folgenden Voraussetzungen:

- Die Parteien des Anschlussvertrages und des Rahmenvertrages kommen überein, die in Art. 11 Abs. 1 des Anschlussvertrages enthaltene Verpflichtung auf den Tiers garant vor Ablauf der Vertragsdauer durch gegenseitige Übereinkunft aufzuheben.

- Versicherungen, die den Wechsel generell zulassen wollen, müssten den Rahmenvertrag einseitig kündigen. Hierbei ist zu beachten, dass Art. 18 Abs. 1 des Rahmenvertrages eine Kündigung frühestens auf das Ende des 18. Monats nach Vertragsbeginn zulässt, wobei eine 6 monatige Kündigungsfrist $\mathrm{zu}$ beachten wäre. Danach kann jeweils mit 6 Monaten Kündigungsfrist auf das Ende eines Kalenderjahres gekündigt werden. Im Kanton Bern dürfen Versicherungen mithin frühestens ab 1. Juli 2005 den Tiers payant anbieten - und auch das nur gegen den Nachweis, dass sie den Anschlussvertrag/Rahmenvertrag rechtzeitig gekündigt haben. Bisher sind bei der Ärztegesellschaft des Kantons Bern keine Kündigungen eingegangen.

- Versicherungen, die den Wechsel zum Tiers payant im Einzelfall zulassen wollen, müssen zusammen mit der jeweiligen Ärztin, dem jeweiligen Arzt den ordentlichen Rücktritt vom Anschlussvertrag/Rahmenvertrag erklären. Ein solcher Rücktritt (Einzelkündigung) kann ebenfalls nur unter Einhaltung einer 6monatigen Frist jeweils per 30. Juni und per 31. Dezember des Kalenderjahres erklärt werden (Art. 4 Anschlussvertrag/Rahmenvertrag). Besonders wichtig für den Kanton Bern: Nach Art. 4 Abs. 1 Anschlussvertrag ist ein Rücktritt «vor Ablauf der Kostenneutralitätsphase» ausgeschlossen. 
Auf das Angebot von MediData kann deshalb nur und erst eingegangen werden, wenn mit Bezug auf den interessierten Arzt der Rahmenund Anschlussvertrag ordnungsgemäss aufgelöst ist oder eine einvernehmliche Anpassung des Vertragswerks zwischen FMH/BEKAG und santésuisse stattgefunden hat. Ein Ausstieg aus dem System des Tiers garant vor Ende der Kostenneutralitätsphase ist nicht nur nicht erwünscht, sondern für die dem bernischen Anschlussvertrag unterstehenden Versicherer und Ärztinnen bzw. Ärzte auch nicht statthaft.

Ärzte, die ohne Einzelkündigung des Rahmen- und Anschlussvertrages vor Ablauf der Kostenneutralitätsphase im Tiers payant abzurechnen beginnen, handeln nach dem Gesagten vertragswidrig.

Nun zu den ökonomischen Überlegungen: Im Tiers payant sieht vordergründig alles einfacher aus. Die Rechnungen können aus den Praxen direkt an die Versicherer geschickt werden - und werden umgehend bezahlt. So verspricht es die einschlägige Werbung. Was ist sonst noch alles zu bedenken?

\section{Der Datenschutz ist im Tiers payant ungenügend}

Der eidgenössische Datenschutzbeauftragte (EDSB) hat diesbezüglich klar Stellung bezogen. Aus Sicht des Datenschutzgesetzes ist der Tiers payant im KVG nur mit expliziter Zustimmung der Patientinnen und Patienten möglich. Der EDSB rät jedoch explizit davon ab, da die Detaillierung der TARMED-Positionen und die Pflicht zur Angabe des Diagnosecodes an die Versicherungen höchst problematisch sind.

\section{Der Patient hat Anrecht auf eine Rechnungskopie}

Gemäss KVG ist dem Patienten im Tiers payant eine Kopie der Arztrechnung zuzustellen. Dies ist - wenn auch in der Praxis oft nicht respektiert - eine gesetzlich zwingende Vorschrift. Damit soll auch der Patient selber überprüfen können, ob die Abrechnung den beanspruchten Leistungen entspricht.

Wenn sich eine Ärztin für den Tiers payant entscheidet, muss sie die Kosten für die Patientenkopien berücksichtigen. Nun ist uns zu Ohren gekommen, dass ein Arzt, welcher mit dem MediPort im Tiers payant abrechnet, sich damit begnügt, in seinem Wartezimmer ein Plakat aufzuhängen, worauf er seine Patienten auffordert, «bei Bedarf» doch eine Rechnungskopie bei der Praxisassistentin zu verlangen ... Sparen auf dem Buckel des Patienten?

\section{Die Kassen honorieren den Tiers payant schlechter}

Die Kassen bevorzugen - trotz des Zinsverlusts wegen der kürzeren Zahlungsfrist - den Tiers payant. Welche Nachteile handeln sich die Praxen damit ein?

- Sie nehmen ein Klumpenrisiko bezüglich Liquidität der Praxis in Kauf: Wenige Kassen machen $80 \%$ des Umsatzes aus. Es reichen eine oder zwei «Zahlungsverzögerungen», um die Praxis in ernsthafte finanzielle Engpässe zu führen. Beispiele für technisch oder auch politisch bedingte Verzüge gibt es in mehreren Regionen und Berufsverbänden.

- Sie geben den Kassen die einmalige Chance, die Rückweisung von Rechnungen direkt mit ihnen abzuwickeln. Der für die Versicherer störende Umweg über die Versicherten findet nicht statt. Damit fällt für die Kassen eine natürliche Hemmung weg, die Rückweisungsquote tief zu halten.

- Sie riskieren, für ihre Leistungen einen tieferen Taxpunktwert zu kassieren - und das trotz elektronischer Anlieferung. So müssen die Ärzte im Kanton Waadt schon heute eine Konzession von 5 Rappen für die Abrechnung im Tiers payant in Kauf nehmen. Bei einem mittleren Rechnungsbetrag von etwa Fr. 250.- macht das über 15 Franken je Rechnung aus!

\section{Die Daten gelangen via MediPort nicht korrekt in den Praxisspiegel}

MediData bietet ihren Kunden gegen einen Aufpreis von 10 Rappen pro Rechnung an, die Daten an ein TrustCenter nach deren Wahl weiterzuleiten. Unsere Erfahrungen zeigen nun, dass die Spezifikationen von TrustX von MediData nicht erfüllt werden.

Die weitergeleiteten Daten sind nicht korrekt codiert und entsprechen auch nicht einer Kopie der Rechnungsdaten. Insbesondere wird der Statistikcode der Patienten falsch berechnet, so dass diese Ärztinnen und Ärzte falsche Patientenzahlen im Praxisspiegel sehen.

Wir empfehlen der Ärzteschaft daher, auf einen Systemwechsel zu verzichten. Für Fragen im Zusammenhang mit dieser Thematik stehen die Autoren gerne zur Verfügung. 